\title{
Nivolumab Regimen
}

National Cancer Institute

\section{Source}

National Cancer Institute. Nivolumab Regimen. NCI Thesaurus. Code C160093.

An immunotherapy regimen consisting of nivolumab that may be used in the treatment

of anal carcinoma; chronic lymphocytic leukemia (CLL); small lymphocytic lymphoma

(SLL); bladder, hepatobiliary, colorectal, kidney, and lung cancers; and cutaneous melanoma. 